University of Nebraska - Lincoln

DigitalCommons@University of Nebraska - Lincoln

Nanoscale insight into the statics and dynamics of polarization behavior in thin film ferroelectric capacitors

Alexei Gruverman

University of Nebraska-Lincoln, agruverman2@unl.edu

Follow this and additional works at: https://digitalcommons.unl.edu/physicsgruverman

Part of the Physics Commons

Gruverman, Alexei, "Nanoscale insight into the statics and dynamics of polarization behavior in thin film ferroelectric capacitors" (2009). Alexei Gruverman Publications. 55.

https://digitalcommons.unl.edu/physicsgruverman/55

This Article is brought to you for free and open access by the Research Papers in Physics and Astronomy at DigitalCommons@University of Nebraska - Lincoln. It has been accepted for inclusion in Alexei Gruverman Publications by an authorized administrator of DigitalCommons@University of Nebraska - Lincoln. 
Published in Journal of Materials Science 44 (2009), pp. 5182-5188; doi: 10.1007/s10853-009-3623-4

Copyright $\odot 2009$ Springer Science+Business Media, LLC. Used by permission.

Submitted April 29, 2009; accepted May 19, 2009; published online June 2, 2009.

FERROELECTRICS

\title{
Nanoscale insight into the statics and dynamics of polarization behavior in thin film ferroelectric capacitors
}

\author{
Alexei Gruverman \\ Department of Physics and Astronomy, University of Nebraska-Lincoln, Lincoln, NE 68588-0111, USA; \\ e-mail alexei_gruverman@unl.edu
}

\begin{abstract}
In this study, we review recent advances in PFM studies of micrometer scale ferroelectric capacitors, summarize the experimental PFM-based approach to investigation of fast switching processes, illustrate what information can be obtained from PFM experiments on domains kinetics, and delineate the scaling effect on polarization reversal mechanism. Particular attention is given to PFM studies of mechanical stress effect on polarization stability.
\end{abstract}

\section{Introduction}

A physical principle of most of ferroelectric-based devices is electrically-induced polarization reversal, which on a microscopic level occurs via the nucleation and growth of a large number of domains. The dynamic characteristics of domain growth as well as static properties of domain structure to a large extent determine the ferroelectric device performance. Recent advances in the synthesis and fabrication of micro- and nanoscale ferroelectric structures [1-4] make it imperative to understand the domain switching behavior at this scale. A major limitation in acquiring this crucial information is the lack of experimental methods to characterize the domain kinetics with the nanometer length and nanosecond time resolution. The most effective approach to visualization of domain kinetics is based on linear coupling between ferroelectric and piezoelectric parameters, which on the experimental level can be detected either by X-ray scattering or by scanning force microscopy. High-resolution studies using time-resolved X-ray microdiffraction im- aging [5-7] have demonstrated reproducible switching behavior of polarization from cycle to cycle and allowed direct measurements of domain wall velocity at high electric fields.

Over the last several years majority of the polarization imaging studies in ferroelectric materials are done by piezoresponse force microscopy (PFM). Application of PFM provided a breakthrough in understanding the static and dynamic behavior in ferroelectric films $[8,9]$. One of the special features of PFM is its capability to detect the polarization state through the top electrode [1012], which provides a unique possibility of nanoscale studies of the statics and dynamics of domain structure in ferroelectric capacitors under the uniform field conditions. This study reviews recent advances in this area of ferroelectric research.

\section{Experimental approach}

In conventional PFM studies of ferroelectrics, a periodic bias, $V_{\text {tip }}=V_{0} \cos \omega t$, with an amplitude $V_{0}$ well below the coercive voltage is applied to a conductive probing tip in contact with a bare surface of the sample, i.e., without a deposited top electrode. Due to the converse piezoelectric effect, application of the ac bias results in a local surface displacement, $d=d{ }_{0} \cos (\omega t+\varphi)$. Domain imaging is performed by detecting the piezoelectric strain using the same tip. A nanoscale dimension of the tipsample contact determines a high spatial resolution of domain features [13]. Due to a highly localized electric field of the tip, application of the bias above the switching threshold results in local polarization reversal and formation of a single nanoscale domain (Figure 1a). 

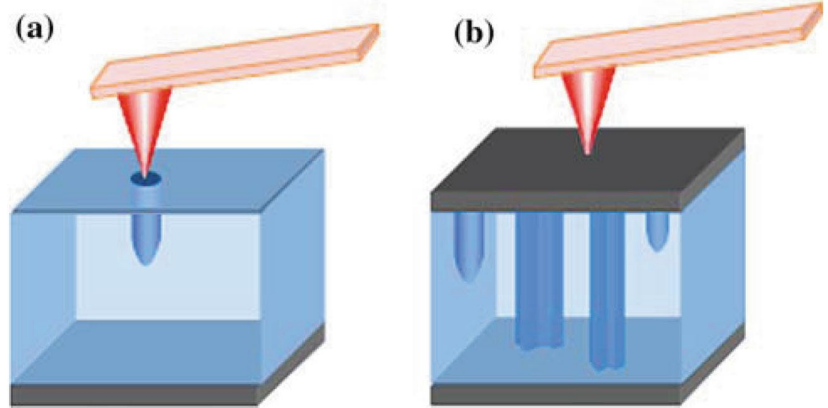

Figure 1. Sketch of domain switching in a standard PFM geometry, and b ferroelectric capacitor structure.

In PFM imaging of the ferroelectric capacitors, the probing tip is in contact with the deposited top electrode (Figure $1 b$ ). Although in this case the whole volume underneath the electrode is electrically excited, the electromechanical response is still probed locally. Scanning the electrode surface while measuring the local strain provides spatially resolved information on domain structure underneath the electrode. The lateral resolution, determined as the domain wall image profile, linearly scales with the thickness of the ferroelectric layer $H$ and top electrode $L$. For typical material parameters and thin top electrode $(L<<H)$, the resolution is expected to be $w \sim 0.2 \cdot H$, which presents the ultimate limit on PFM resolution in capacitors [14].

External bias applied to the top electrode generates a uniform electrical field within the capacitor so that the PFM tip senses the response from the whole thickness of the ferroelectric layer. Thus, this approach allows one to get around the problem of an inhomogeneous field distribution generated by the probing tip in a film without a top electrode. Additionally, domain imaging in capacitors provides a possibility of direct studies of polarization reversal dynamics, which on a microscopic level occurs via the nucleation and growth of a large number of domains (Figure 1b). This PFM-assisted insight into the domain kinetics has significant advantage over conventional electrical testing of the switching behavior as it allows direct assessment of the relative contribution of nucleation and domain wall motion into polarization reversal process, field-dependent motion of domain walls and capacitor size effect on its switching behavior.

Generally, application of PFM to investigation of dynamic processes is limited by its low time resolution determined by acquisition time of a single frame (of the order of several minutes). A high-speed version of PFM (HSPFM) has been developed by Huey's group to allow image acquisition in several seconds effectively increasing time resolution by two orders of magnitude over the conventional PFM imaging $[15,16]$. This approach, which involves high-speed scanning of a bare ferroelectric surface with a tip under a superposition of a switch- ing and imaging bias, allows effective studies of the dynamics of domain nucleation and growth but requires relatively smooth surfaces.

Recently, time resolution of PFM imaging has been improved even further (into the $100 \mathrm{~ns}$ range) by using an interrupted switching PFM (IS-PFM) method based on visualization of domain configurations developing in ferroelectric capacitors during step-by-step polarization reversal [17]. Switching characteristics such as nucleation rate and domain wall velocity can be calculated from a set of PFM snapshots taken at different time intervals by measuring the time dependence of the number and size of growing domains. The time resolution of the IS-PFM method is determined by the rise time and duration of the switching pulses and, depending on the capacitor size and time constant of the external circuitry, can be in the order of $10 \mathrm{~ns}$.

Two main modifications of the IS-PFM method have been suggested (Figure 2). In one approach, the domain switching behavior is visualized by applying a series of short input pulses with fixed amplitude and incrementally increasing duration $\left(\tau_{1}<\tau_{2}<\ldots<\tau_{n}<t_{s} \tau<t_{s}\right.$, where $t_{s}$ is a switching time for a given voltage). PFM imaging of the resulting domain pattern is performed after each pulse (Figure 2a) [18]. At the beginning of each switching cycle, the capacitor is reset into the initial polarization state. Applicability of this approach depends upon the reproducibility of domain switching kinetics from cycle to cycle. Indeed, it has been shown, that in each switching cycle, domain nucleation occurs in the predetermined sites most likely corresponding to the local defects at the film-electrode interface. Figure 3 shows

(a)

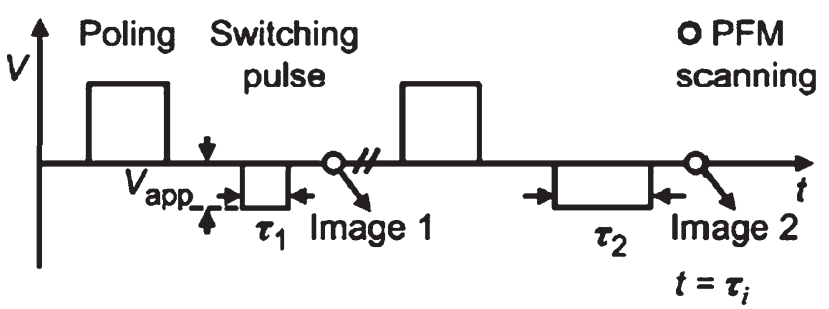

(b)

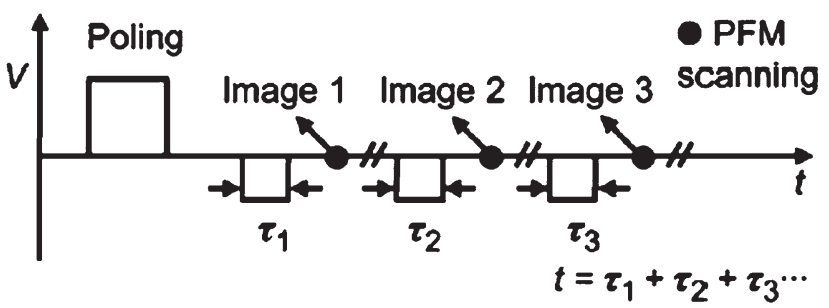

Figure 2. Schematic of pulse sequences for IS-PFM imaging of domain switching kinetics: a) step-by-step switching approach, b) successive switching approach. Reprinted with permission from [20]. 


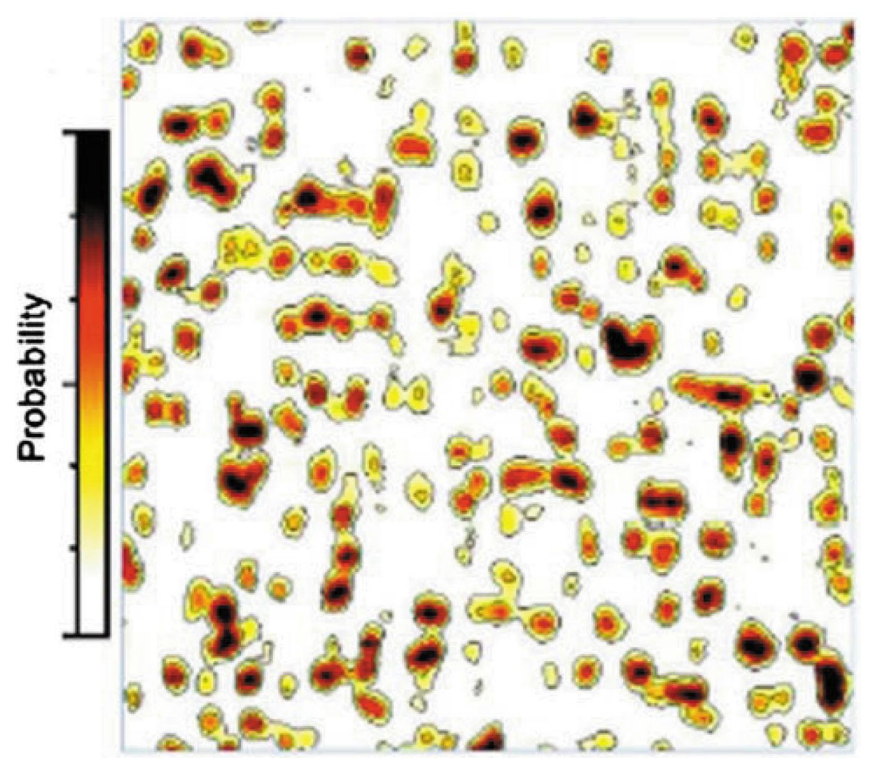

Figure 3. A two-dimensional map of nucleation probability acquired in epitaxial PZT capacitor. The scan area is a $6 \times 6 \mu \mathrm{m}^{2}$. Reprinted with permission from [29].

a two-dimensional map of on nucleation probability obtained by Kim et al. [19] in epitaxial $\mathrm{Pb}(\mathrm{Zr}, \mathrm{Ti}) \mathrm{O}_{3}$ (PZT) capacitors. This map is a direct illustration of the fact that ferroelectric switching is a result of heterogeneous nucleation. Most sites in epitaxial capacitors have nucleation probability above $90 \%$ while in polycrystalline capacitors probability is close to $100 \%$.

In another approach, proposed to reduce the detrimental effect of stochastic nucleation events in epitaxial structures, after a capacitor is being set, the switching pulses of the same duration are applied to the capacitor $\left(\tau_{1}=\tau_{2}=\ldots=\tau_{n}<t_{s}\right)$ with PFM imaging between the pulses (Figure 2b) [20]. In this case, it is assumed that the PFM image obtained after the $n$-th pulse is the same as that after a single pulse with duration of $t=\tau_{1}+\tau_{2}+\ldots+\tau_{n}$. Then, all the PFM images taken be- fore the $(n+1)$-th pulse reveal the successive domain wall evolution during the time period of $t$.

It should be mentioned that both variations of the ISPFM method rely on stability of instantaneous domain patterns between pulse applications. Whether polarization relaxation takes place or not can be checked by comparing the PFM switching with the transient current measurements. Little or no discrepancy between the two sets of data obtained in most reports is a solid proof of the reliability of the IS-PFM approach. A small dc offset during PFM imaging can be used to account for an internal bias (imprint) in the capacitors and stabilize the domain patterns [21].

\section{Capacitor scaling effect on domain switching kinetics}

Application of the IS-PFM approach to fast switching processes (in the 100-ns range) allowed identification of the effect of capacitor size on the rate-limiting mechanism. Figure 4 shows PFM images of instantaneous domain configurations developing in $1 \times 1.5$ and $5 \times 5 \mu^{2}$ polycrystalline (111) PZT capacitors at different stages of polarization reversal process [18]. IS-PFM switching data in Figure 4 indicate the difference in switching mechanisms in larger and smaller capacitors. Generally, in larger capacitors, two distinct stages of polarization reversal can be observed: a fast switching stage dominated by domain nucleation (usually up to $60-70 \%$ of the total volume) and a slower stage where switching occurs mainly via lateral domain wall motion. On the other hand, in the small capacitors the switching proceeds mostly via lateral growth of just few domains, i.e., contribution of domain nucleation to polarization reversal is significantly reduced. Due to the qualitatively different domain dynamics, the relative switching speed of capacitors of smaller dimensions is field dependent: they switch faster than the larger capacitors in the high field range (in agreement with earlier reports by macroscopic measurements [22]), but slower in the low fields (Figure 5a) [18].
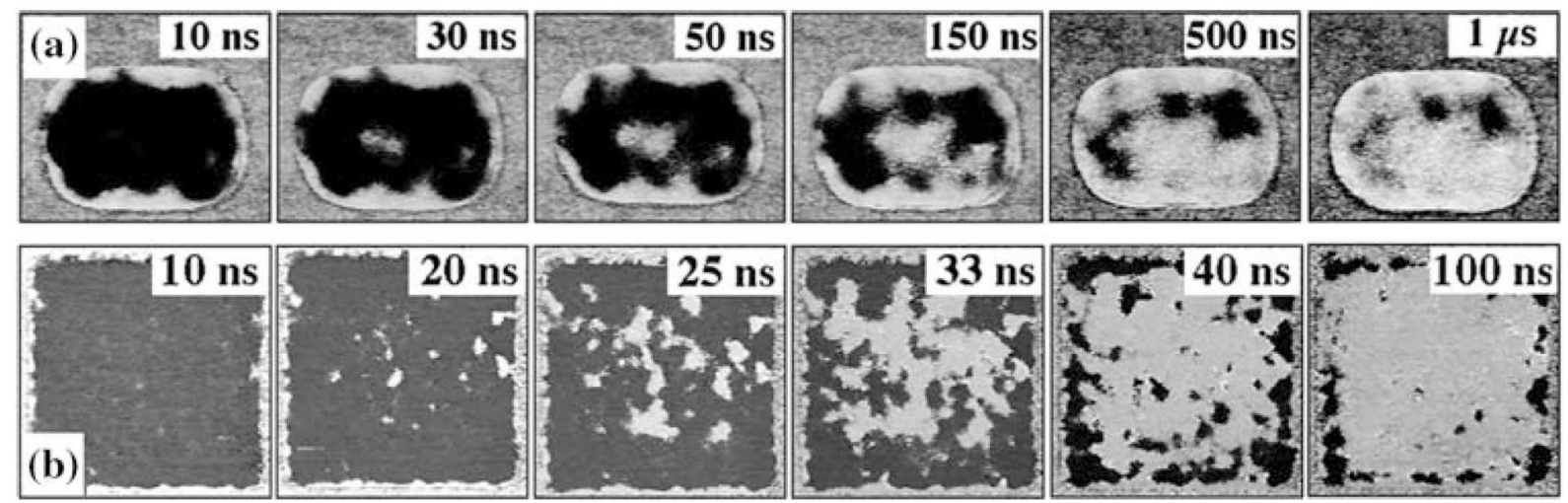

Figure 4. PFM phase images of domain configurations developing in polycrystalline (111) PZT capacitors, a) $1 \times 1.5 \mu m^{2}$ and $\mathbf{b}$ ) $5 \times 5 \mu \mathrm{m}^{2}$, at different stages of step-by-step polarization reversal in the low field range $(E=100 \mathrm{kV} / \mathrm{cm})$. The scanning area is $1.6 \times 1.6 \mu \mathrm{m}^{2}$ in (a) and $5.5 \times 5.5 \mu \mathrm{m}^{2}$ in (b). Reprinted with permission from [28]. 

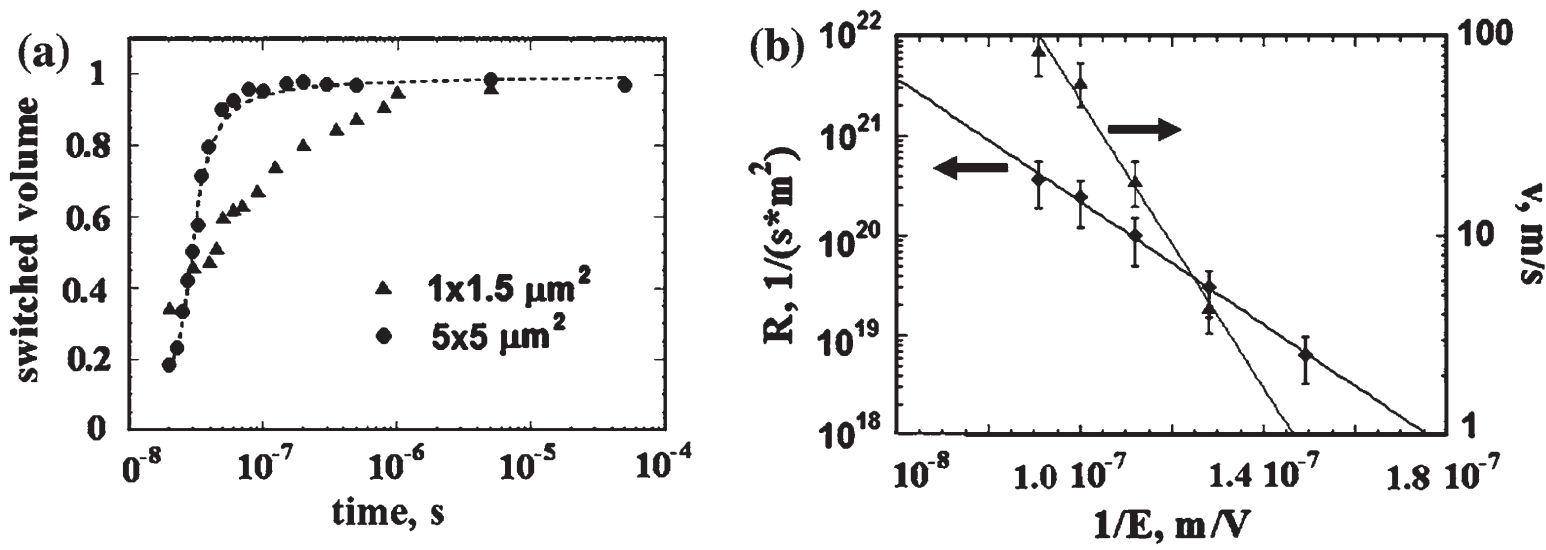

Figure 5. a) Switched capacitor volume as a function of time for $1 \times 1.5$ and $5 \times 5 \mu \mathrm{m}^{2}$ (111) PZT capacitors in the low field range $(E=100 \mathrm{kV} / \mathrm{cm})$ showing faster switching in larger capacitors. Smaller capacitors start to switch faster in the fields above $200 \mathrm{kV} /$ $\mathrm{cm}$. Dashed line-fitting of the PFM switching data by the NLS model using Lorentzian distribution for log $t_{0}$. $\mathbf{b}$ ) Nucleation rate and domain wall velocity vs. electrical field for $5 \times 5 \mu^{2}$ capacitors. Reprinted with permission from [28].

Quantitative insight into the capacitor scaling effect on switching behavior has been obtained by measuring the field dependency of nucleation rate $R$ and wall velocity $v$ (Figure $5 b$ ) obtained from the time dependence of domain number and domain radius, respectively [17]. It is found that both parameters are exponential functions of the applied field: $R \sim \exp \left(-a_{n} / E\right)$ and $v \sim \exp (-a$ $\left.{ }_{w} / E\right)$, where $a_{n}=7.1 \times 10^{7} \mathrm{~V} / \mathrm{m}$ and $a_{w}=8.3 \times 10^{7} \mathrm{~V} /$ $\mathrm{m}$. A difference between activation field values $a_{n}$ and $a$ ${ }_{w}$ in combination with the capacitor size-dependent polarization switching mechanism explains faster switching in $1 \times 1.5 \mu \mathrm{m}^{2}$ capacitors in comparison to $5 \times 5 \mu \mathrm{m}^{2}$ capacitors in the high field range by virtue of transition from wall-limited to nucleation-limited switching. As contribution of nucleation to the polarization switching in smaller capacitors is insignificant (they switch mainly via the domain wall motion), in the high field range, where nucleation is a rate-limiting mechanism, the smaller capacitors will switch faster than the larger capacitors. In the low-field range, on the contrary, domain wall speed will limit the switching rate. For this reason, larger capacitors characterized by considerable contribution of nucleation mechanism to polarization reversal will be switching faster in low fields. Theoretical estimations show that the nucleation time becomes a rate-limiting parameter for polycrystalline PZT capacitors smaller than $0.6 \mu^{2}$ in the fields above $200 \mathrm{kV} / \mathrm{cm}$ [18].

\section{Effect of film microstructure on domain switching kinetics}

Direct imaging of domain evolution during switching provides a possibility to understand the effect of film microstructure on polarization reversal behavior. A traditional approach to model the switching kinetics - the KAI model-is based on the classical statistical theory of nucleation and unrestricted wall motion proposed by Kolmogorov and Avrami, and extended to ferroelectrics by Ishibashi [23]. In the KAI model, the volume fraction of the polarization switched by time $t$ is described by $p(t)=1-\exp \left[-\left(t / t_{0}\right)^{n}\right]$, where $t_{0}$ is the characteristic switching time and $n$ can be treated as domain dimensionality reflecting the mechanism of polarization reversal. The KAI model has been successfully applied to the switching in epitaxial thin films and single crystals. However, switching in polycrystalline films proceeds much slower than predicted by the KAI model [24] and continuous domain wall motion is presumably interrupted due to pinning by structural defects. Validity of this assumption has been confirmed by IS-PFM observation of spatial variations in domain wall velocity by almost two orders of magnitude [10]. It has been also shown that the KAI model cannot adequately fit the domain switching kinetics. A nucleation-limited (NLS) model featuring independent switching kinetics in individual grains and an exponentially wide variation of local nucleation times has been proposed to explain switching in polycrystalline capacitors $[25,26]$. The time-dependent behavior of switched polarization is expressed by $p(t)=1-\sum F(\log$ $\left.t_{0}\right) \exp \left(-\left(t / t_{0}\right)^{n}\right)$, where $F\left(\log t_{0}\right)$ is a distribution function for local switching times. To account for variations in the local electrical field related to the randomly distributed dipole defects, Jo et al. [27] suggested to use the Lorentzian distribution of $\log t_{0}$. This treatment allows description of the switching current data within a wide range of electric fields and temperatures. IS-PFM measurements show that this approach provides the best fit for experimentally observed domain kinetics in relatively large polycrystalline PZT capacitors (Figure 5a). In contrast, the switching kinetics in smaller capacitors is close to the logarithmic time dependence, which can be a result of a much narrower distribution of $\log t_{0}$.

Snapshots of domain structure evolution during switching in epitaxial (001) PZT capacitors are shown 
in Figure 6. Note that the switching as a whole occurs via dynamics of $180^{\circ}$ domains and no $90^{\circ}$ wall formation has been observed in agreement with earlier conclusion based on transient current measurements [28]. For $E=700 \mathrm{kV} / \mathrm{cm}$, the nucleation density was estimated to be $7.1 \times 10^{8} \mathrm{~cm}^{-2}$ (Figure 6a), which is well below the nucleation density of $3.2 \times 10^{12} \mathrm{~m}^{-2}$ measured in polycrystalline PZT capacitors [18]. Given a higher concentration of point defects associated with grain boundaries and relatively rough interfaces in polycrystalline films, this difference is reasonable. The time-dependent evolution of domain structure reveals that the wall velocity is isotropic and independent of domain size in the range at least up to $400 \mathrm{~nm}$ in diameter. Considerable decrease in the wall velocity has been observed for domains in the close proximity $(<100 \mathrm{~nm})$ to another growing domains.

Significant anisotropy of the wall velocity has been detected during switching in the lower field range $(<500 \mathrm{kV} / \mathrm{cm})$. This anisotropy is manifested by the formation of characteristically elongated domains (Figure $6 \mathrm{~b}$ ). Measurements of domain size as a function of time show that in the $y$-axis direction the wall velocity $v_{y}$ is almost three times higher than velocity $v_{x}$ in the orthogonal $x$-axis direction $(0.39 \mathrm{~m} / \mathrm{s}$ and $0.12 \mathrm{~m} / \mathrm{s}$, respectively). Overall, the switching kinetics in the low fields is characterized by a long-range (up to $1 \mu \mathrm{m}$ ) lateral growth of just a few nucleated domains in stark contrast to the switching in the high fields.

For epitaxial capacitors, the KAI model provides excellent description of the time-dependent switching behavior, which in stark contrast with the case of polycrystalline PZT capacitors. It has been found that $n$ values are different for low and high fields and very close to integer values: 1.12 for $500 \mathrm{kV} / \mathrm{cm}$ and 1.96 for $700 \mathrm{kV} /$ $\mathrm{cm}$, indicating a change in the switching mechanism. In the KAI model, $n=2$ corresponds to a growth of cylindrical domains (2D growth) and $n=1$ indicates a lateral expansion of lamellar domains (1D growth). An increase in $n$ with an applied field, also reported by So et al. [29], is consistent with the change in domain growth dimensionality revealed by IS-PFM studies - transition from one-dimensional anisotropic growth under $500 \mathrm{kV} / \mathrm{cm}$ to two-dimensional isotropic growth under $700 \mathrm{kV} / \mathrm{cm}$.

A general approach adopted by Noh et al. [30] treats domain wall motion as a nonlinear dynamic process resulting from competition between elastic and pinning forces. Investigations of domain growth in a wide range of temperature and electric field showed that the wall dynamics could be classified into the creep, depinning, and flow regimes. Wall velocity exhibits high temperature dependence in low fields, while in high fields a crossover to the flow regime makes it temperature independent. In the creep regime at low fields, the wall dynamics can be described as thermally activated propagation between pinning sites: $v \sim \exp \left[-\left(U / k_{B} T\right)\left(E_{c} / E\right)^{\mu}\right]$,
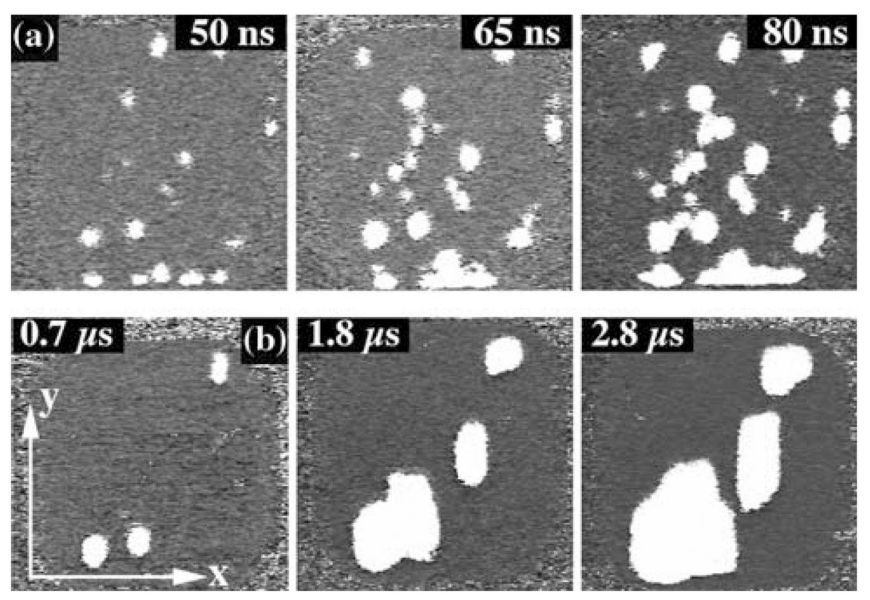

Figure 6. PFM phase images of domain configurations developing in epitaxial (001) PZT capacitors a $E=700 \mathrm{kV} / \mathrm{cm}$; b $E=500 \mathrm{kV} / \mathrm{cm}$. The scan size is $2.5 \times 2.5 \mu^{2}$.

where $U$ is an energy barrier and $\mu$ is a dynamic exponent, reflecting the nature of the pinning potential. It was found that for epitaxial PZT capacitors $\mu=0.9 \pm 0.1$ over a wide temperature range suggesting a long-range pinning potential due to defect structure.

\section{Mechanical stress effect on static polarization behavior}

The static polarization state in ferroelectric capacitors, determined by the thermodynamic minimum of free energy, is a function of electrical and mechanical boundary conditions. Reduction of the free energy via formation of ferroelectric domain structures gives rise to a profound mechanical stress effect on the physical properties of ferroelectrics. Accommodation of the misfit strain between a substrate and a thin film in epitaxial heterostructures can result in the appearance of new phases forbidden in bulk samples and significant size dependence on the dielectric and piezoelectric properties [31]. Numerous attempts have been made to develop a thermodynamic theory that would account for the effect of the mechanical boundary conditions on structural transformations in ferroelectric films [32-34]. However, up to now almost all of these studies took into account the mechanical boundary conditions only at the film/substrate interface while effect of the mechanical stress imposed by the top electrode (TEL) has largely been ignored. Application of PFM reveals that boundary conditions at the top interface drastically affect the polarization stability in polycrystalline PZT capacitors.

Figure 7 shows surface topography, PFM amplitude, and phase images of the poled $1 \times 1.5 \mu^{2}$ capacitors with a 50-nm thick TEL [35]. Capacitors in the upper row have been poled by $-5 \mathrm{~V}, 1 \mathrm{~s}$ voltage pulses applied to the top electrodes, while the bottom row capacitors have 
Figure 7. a) Topographic, b) PFM phase, and c) PFM amplitude of the poled (111) PZT capacitors with 50nm thick top electrodes. Upper row capacitors poled by negative voltage pulses $(-5 \mathrm{~V}, 1 \mathrm{~s})$, bottom row capacitors poled by positive pulses $(+5 \mathrm{~V}, 1 \mathrm{~s})$. The scanning size is $6 \times 6 \mu \mathrm{m}^{2}$. Reprinted with permission from [35].

Figure 8. a) Topographic, b) PFM phase, and c) PFM amplitude of the poled (111) PZT capacitors with 250-nm thick top electrodes. Upper row capacitors poled by negative voltage pulses $(-5 \mathrm{~V}, 1 \mathrm{~s})$, bottom row capacitors poled by positive pulses $(+5 \mathrm{~V}, 1 \mathrm{~s})$. The scanning size is $6 \times 6 \mu \mathrm{m}^{2}$. Reprinted with permission from [35].

Figure 9. Finite element phase field map of the out-of-plane (111) polarization component $\left(\mathrm{P}_{2}\right)$ in two short-circuited ferroelectric capacitors with a lateral size of $1 \mu \mathrm{m}$ under equilibrium conditions: a) 250nm thick TEL; b) 50-nm thick TEL. Reprinted with permission from [35].
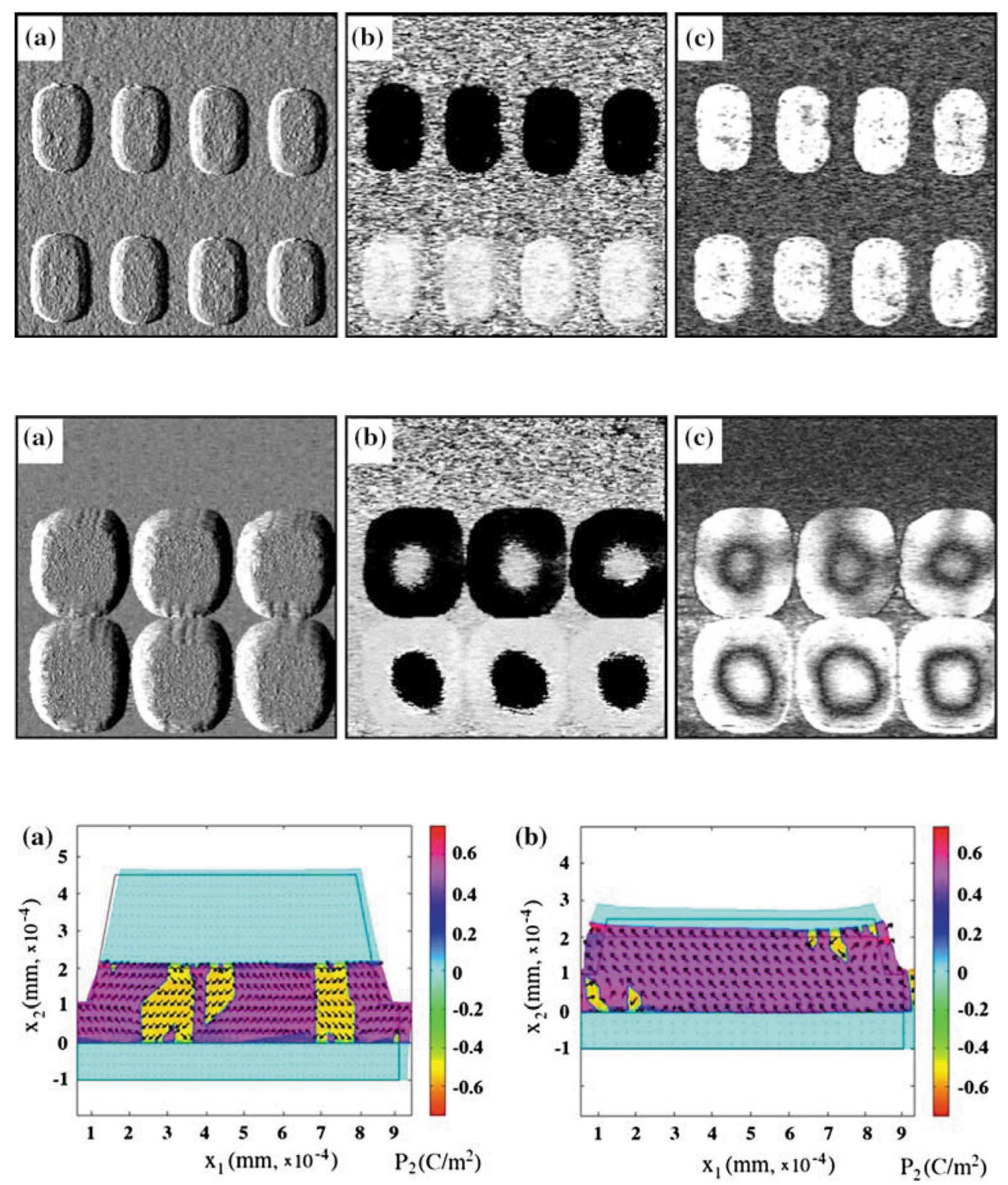

been poled into opposite direction by the $+5 \mathrm{~V}, 1 \mathrm{~s}$ voltage pulses. Uniform amplitude and phase contrast indicate complete and uniform switching of the capacitors into a stable polarization state.

On the other hand, PFM imaging of the poled capacitors with the 250-nm thick TEL (Figure 8) reveals unusual domain patterns: after application of poling voltage the central regions of the capacitors exhibit polarization opposite to the polarity of the applied voltage. The observed effect is symmetric with respect to the voltage polarity, i.e., the central parts of the capacitors always exhibit polarization opposite to the applied bias. It should be mentioned that imaging the same capacitors in the PFM mode with an additional dc bias superimposed on the ac imaging voltage resulted in complete switching of polarization in the whole capacitor indicated by a uniform PFM signal across the top electrode. However, after the dc bias is turned off, the inverse domain in the center region appears again. This behavior is indicative of spontaneous backswitching occurring in the center of the capacitors after application of the poling voltage. These results also suggest that the back-switching effect in the PZT capacitors is not affected by the substrate but is the result of the presence of a thick (250 nm) top electrode.

Free energy calculations using a fully-coupled ferroelectric finite element phase field (FFEP) model [36] show mechanical constraints due to the thick top electrode create residual shear stresses during ferroelectric switching in (111) PZT capacitors leading to complex, twinned domain structure (Figure 9). The residual elastic energy results in spontaneous polarization backswitching in the central regions of capacitors. Residual elastic energy is larger in the center away from the stress-free edges, which explains why backswitching mainly occurs in the central parts of the capacitors.

Also, recently residual stress has been suggested to cause faceting in poled epitaxial (001) PZT capacitors [37]. 


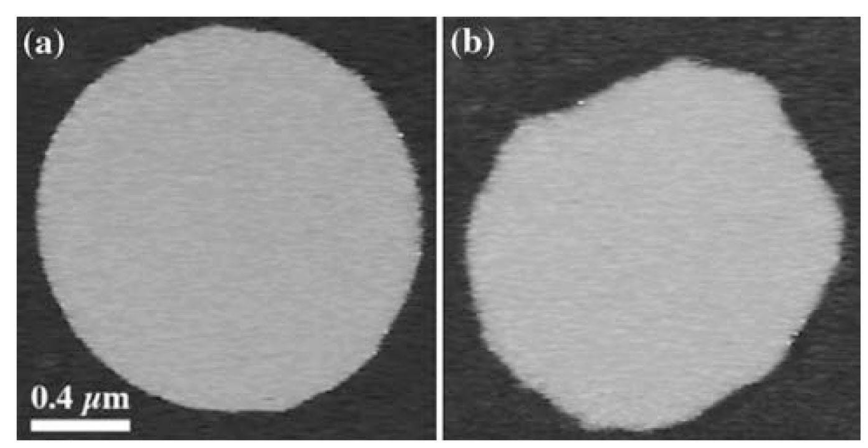

Figure 10. PFM phase images of the poled circular epitaxial PZT capacitor a) right after poling, and b) $24 \mathrm{~h}$ after poling. Reprinted with permission from [37].

It has been shown that within a period of time much longer than the switching time the metastable circular domains develop facets along (100) directions (Figure 10). Faceting can be attributed to anisotropic contribution to the wall velocity along the crystallographic axes [38]. The observed effect is interpreted as mechanical relaxation resulting from highly inhomogeneous stress distribution in the circular capacitors with high-stress conditions in the center due to electrode-film lattice mismatch and presumably stress-free edges.

\section{Conclusion}

Rapid development of ferroelectric-based devices with reduced dimensions generated a strong need for extensive investigation of the size effects in ferroelectric materials. Application of PFM provides a unique opportunity to study physical mechanisms underlying the static and dynamic properties of ferroelectric structures at the nanoscale level. Inhomogeneous domain nucleation results in reproducibility of switching kinetics and allows application of the interrupted switching PFM approach to study fast polarization reversal processes. Direct measurements of the field-dependent wall velocity and nucleation rate provide information on the mechanism of domain growth in conjunction with microstructural and scaling effects.

\section{Acknowledgements}

This study was supported by the National Science Foundation (Grant Nos. MRSEC DMR-0820521) and the Nebraska Center for Materials and Nanoscience at University of Nebraska-Lincoln. The author would like to thank Prof. T. W. Noh for his kind permission to use his data.

\section{References}

1. Ganpule CS, Stanishevsky A, Aggarwal S, Melngailis J, Williams E, Ramesh R, Joshi V, de Araujo CP (1999) Appl Phys Lett 75:3874

2. Yun WS, Urban JJ, Gu Q, Park H (2002) Nano Lett 2:447

3. Luo Y, Szafraniak I, Zakharov ND, Nagarajan V, Steinhart M, Wehrspohn RB, Wendorff JH, Ramesh R, Alexe M (2003) Appl Phys Lett 83:440

4. Morrison FD, Ramsay L, Scott JF (2003) J Phys Condens
Matter 15:L527

5. Grigoriev A, Do D-H, Kim DM, Eom C-B, Adams B, Dufresne E, Evans PG (2006) Phys Rev Lett 96:187601

6. Do DH, Grigoriev A, Kim DM, Eom C-B, Evans PG, Dufresne EM (2008) Integr Ferroelectr 101:174

7. Grigoriev A, Sichel R, Lee H-N, Landahl EC, Adams B, Dufresne EM, Evans PG (2008) Phys Rev Lett 100:027604

8. Alexe M, Gruverman A (eds) (2004) Nanoscale characterization of ferroelectric materials: scanning probe microscopy approach. Springer-Verlag, Berlin

9. Jesse S, Baddorf AP, Kalinin SV (2006) Appl Phys Lett 88:062908

10. Gruverman A, Rodriguez BJ, Nemanich RJ, Kingon AI, Cross JS, Tsukada M (2003) Appl Phys Lett 82:3071

11. Bintachitt P, Trolier-McKinstry S, Seal K, Jesse S, Kalinin SV (2009) Appl Phys Lett 94:042906

12. Hong S, Colla EL, Kim E, Taylor DV, Tagantsev AK, Muralt P, No K, Setter N (1999) J Appl Phys 86:607

13. Tian L, Vasudevarao A, Morozovska AN, Eliseev EA, Kalinin SV, Gopalan V (2008) J Appl Phys 104:074110

14. Kalinin SV, Rodriguez BJ, Kim S-H, Hong S-K, Gruverman A, Eliseev EA (2008) Appl Phys Lett 92:152906

15. Nath R, Chu Y-H, Polomoff NA, Ramesh R, Huey BD (2008) Appl Phys Lett 93:072905

16. Polomoff NA, Nath R, Bosse JL, Huey BD (2009) J Vac Sci Technol B 27:1011

17. Dehoff C, Rodriguez BJ, Kingon AI, Nemanich RJ, Gruverman A, Cross JS (2005) Rev Sci Instrum 76:023708

18. Gruverman A, Wu D, Scott JF (2008) Phys Rev Lett 100:097601

19. Kim DJ, Jo JY, Kim TH, Yang SM, Chen B, Kim YS, Noh TW (2007) Appl Phys Lett 91:132903

20. Yang SM, Jo JY, Kim DJ, Sung H, Noh TW, Lee HN, Yoon J-G, Song TK (2008) Appl Phys Lett 92:252901

21. Gruverman A, Rodriguez BJ, Dehoff C, Waldrep JD, Kingon AI, Nemanich RJ, Cross JS (2005) Appl Phys Lett 87:082902

22. Hase T, Shiosaki T (1991) Jpn J Appl Phys 30:2159

23. Ishibashi Y, Takagi Y (1971) J Phys Soc Jap 31:506

24. Lohse O et al (2001) J Appl Phys 89:2332

25. Du XF, Chen IW (1998) Appl Phys Lett 72:1923

26. Tagantsev A et al (2002) Phys Rev B 66:214109

27. Jo JY, Han HS, Yoon J-G, Song TK, Kim S-H, Noh TW (2007) Phys Rev Lett 99:267602

28. Li W, Alexe M (2007) Appl Phys Lett 91:262903

29. So YW, Kim DJ, Noh TW, Yoon J-G, Song TK (2005) Appl Phys Lett 86:092905

30. Jo JY, Yang SM, Kim TH, Lee HN, Yoon J-G, Park S, Jo Y, Jung MH, Noh TW (2009) Phys Rev Lett 102:045701

31. Pertsev NA, Zembilgotov AG, Tagantsev AK (1998) Phys Rev Lett 80:1988

32. Pertsev NA, Arlt G, Zembilgotov AG (1995) Microelectron Eng 29:135

33. Speck JS, Seifert A, Pompe W, Ramesh R (1994) J Appl Phys 76:477

34. Pompe W, Gong X, Suo Z, Speck JS (1993) J Appl Phys 74:6012

35. Gruverman A, Cross JS, Oates WS (2008) Appl Phys Lett 93:242902

36. Su Y, Landis C (2007) J Mech Phys Solids 55:280

37. Scott JF, Gruverman A, Wu D, Vrejoiu I, Alexe M (2008) J Phys Condens Matter 20:425222

38. Kleemann W, Dec J, Prosandeev SA, Braun T, Thomas PA (2006) Ferroelectrics 334:3 